\title{
Writing SUBROUTINE HOWFAR for EGS4
}

\author{
W. R. Nelson and T. M. Jenkins \\ Stanford Linear Accelerator Center, \\ Stanford, California, 94305, USA
}

\subsection{INTRODUCTION}

The EGS4 Code System ${ }^{1}$ requires that the user write a driver program, most commonly referred to as an EGS User Code. The User Code generally consists of a MAIN routine, SUBRDUTINE AUSGAB, SUBROUTINE KOIFAR, and any auxiliary subprograms that the user may require for the problem at kand. The purpose of MAIN is to set up various initializations and to provide for two necessary subroutine calls:

- CALL HATCH to bring in the various media data created at an earlier tine by PEGS4, and

- CALL SHOWER to initiate the radiation transport process itself, for as many incident events (histories) desired.

SUBROUTINE AUSGAB must be written in order to get results from EGS during the simulation. If the user does not want to score anything, then AUSGAB is trivial. Namuly,

SUBROUTINE AUSGAB (IARG);

RETURN;

END;

SUBROUTINE HOWFAR is required in order to provide information to EGS about the nature of the geometry. Obviously, the most trivial HOWFAR is the case of a homogeneous infinite medium, which is again accomplished very simply with

SUBROUTINE HOWFAR;

RETURN;

END;

Work supported by the US Department of Energy under contract DE- $A$ C03-76SF00515 
The purpose of this note is to provide guidance, in addition to that given in $\mathrm{SJ}, \mathrm{AC}$ 265 , in writing SUBROUTINE HOWFAR for both simple and complex geometrical situations. Since most complex geometries can be represented in terms of ilocks of simpler geometries, a number of geometry subprograms have been created for use within HOWFAR. They are provided as part of the EGS4 Code System, which means that they are located in files on the EGS4 disk under VM/SP at SLAC. They are also on the EGS4 Distribution Tape that is given out on request by the SLAC Radiation Physics Group.

What we hope to accomplish with this note is to show how to create EGS4 geometries in a modular fashion, with particular emphasis on using the macro equivalents of the subroutines in order to gain efficiency. It is assumed that the reader is already famijar with EGS4 and understands the role of the variables USTEP, IDISC, and IRNEW as they apply to SUBRDUTINE HOWFAR. If not, then the reader is advised to study Appendix 2 of SLAC-265 (at least Section A2.5) first. The tutorial chapter (Chapter 3) may also provide some insight.

\subsection{THE EGS4 GEOMETRY SUBROUTINES}

A variety of subprograms, designed to aid the user in creating relatively sophisticated User Codes, are available with the EGS4 Code System*. Each routine listed below is self-documented by means of commentary contajned within the coding.

PLANE1 - Determines if particle trajectory strikes a plane surface. Returns trajectory distance (TPLN).

CYLNDR - Determines if the particle trajectory strikes a cylindrical surface. Returns trajectory distance (TCYL).

CONE - Determines if the particle trajectory strikes a conical surface. Returns trajectory distance (TCON).

SPHERE - Determines if the particle trajectory strikes a spherical surface. Returns trajectory distance (TSPH).

CHGTR - Changes USTEP and IRNEW whenever USTEP is larger than the trajectory distance (TPLN, TCYL, TCON, TSPH).

FINVAL - Determines the coordinates of the particle trajectory at the point of an intersection with a given surface.

PLAN2P - Determines the intersection point for two parallel planes by calling PLANE1 twice (when necessary) and CHGTR if a plane is hit.

PLAN2X - Determines the intersection point for two crossing planes by calling PLANE1 twice (always) and CHGTR if a plane is hit (PLAN2X is slightly less efficient than PLAN2P).

CYL2 - Similar to PLAN2P, but for concentric cylinders.

CON2 - Similar to PLAN2P, büt for concentric cones.

SPH2 -- Similar to PLAN2P, but for concentric spheres.

* See file GEOMAUX MORTRAN (\#26) on the EGS4 Distribution Tape. 


\subsection{SUBROUTINE \& MACRO EXAMPLES}

\subsubsection{Subroutines PLANE1 and CHGTR.}

Assume that we have two parallel planes separating three regions of interest, as shown in Fig. 1.1. In this figure, the regions are identified by the numbers 22,23 and 24 , and the planes by the numbers 6 and 7 . The numbers were chosen arbitrarily, but with a general purpose in mind-to make it somewhat easy for the reader to identify how they are used with PLANE1 and CHGTA. The triangles that enclose the numbers 6 and 7 also have a purpose-they point in the direction of the unit normal vector that is generally initialized in KAIN and passed in COMIN/PLADTA/. Thus, in the case represented by Fig. 1.1,

$\operatorname{PNORM}(1,6)=0.0 ; \operatorname{PNORM}(2,6)=0.0 ; \operatorname{PNORM}(3,6)=1.0 ;$

$\operatorname{PNORM}(1,7)=0.0 ; \operatorname{PNORH}(2,7)=0.0 ; \operatorname{PNORH}(3,7)=1.0 ;$.

To illustrate, for the moment, how one completes the definition of the two planes, let plane 6 be located at $z=30.0$ and plane 7 at $z=45.0$ relative to the origin, $O$. Then

$$
\begin{array}{lll}
\operatorname{PCOORD}(1,6)=0.0 ; & \operatorname{PCOORD}(2,6)=0.0 ; & \operatorname{PCOORD}(3,6)=30.0 ; \\
\operatorname{PCOORD}(1,7)=0.0 ; & \operatorname{PCOORD}(2,7)=0.0 ; & \operatorname{PCOORD}(3,7)=45.0 ;
\end{array}
$$

where the PNORMS and PCOORDs are passed from the MAIN program into subprograms by means of COMIN/PLADTA/ (Note: COMIN/PLADTA/ is expanded out by a macro into COMMON/PLADTA/PCOORD ( $3, \$ M X P L N S)$, PNORH ( $3, \$ M X P L N S)$, where \$NXPLNS is the maximum number of planes (default $=100$ ) determined by the user).

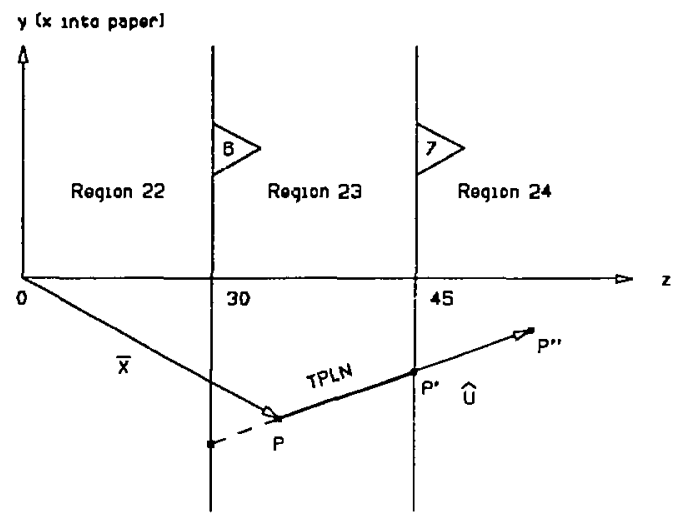

Figure 1.1. Two parallel planes separating three regions along $\mathrm{z}$.

Now, let us assume that the particle currently being transported is located in region 23 at vector position $\overrightarrow{\mathbf{X}}$, traveling in the direction given by unit vector $\hat{U}$, and that this information is available through COMIN/SIACK/. The purpose of SUBROUTINE HOHFAR is 
to determine if the next transport step that EGS4 plans to take, USTEP $\left(=\left|\overrightarrow{P P^{\prime \prime}}\right|\right)$, is such that the particle will be transported out of the current region into another. If this is the case, then the user must reduce USTEP to a value consistent with the boundary and set IRNEH equal to the new region. Upon returning to the calling program (either ELECTR or PHOTON), the EGS4 system will use this information to break the transport into two steps, correctly accounting for possible changes in the media at the boundary.

The purpose of SUBROUTINE PLANE1 is to determine if the plane is intersected by the trajectory (IHIT=1) and to calculate the distance to the intersection, TPLN $\left(=\left|\overrightarrow{P P^{\prime}}\right|\right)$. If the trajectory is parallel to or away from the plane, IHIT takes on the value of 2 or 0 , respectively, and TPLN has no relevance. The purpose of SUBROUTINE CHGTR is to set USTEP=TPLN and and to assign the new region to IRNEW, but only if USTEP $\geq$ TPLN (and provided that IHIT $=1$, of course).

In order to make things as simple as possible, let us assume in our example that particles are to be transported in region 23 , but discarded once they reach regions 22 or 24, as described by the SUBROUTINE HOHFAR shown in Fig. 1.2

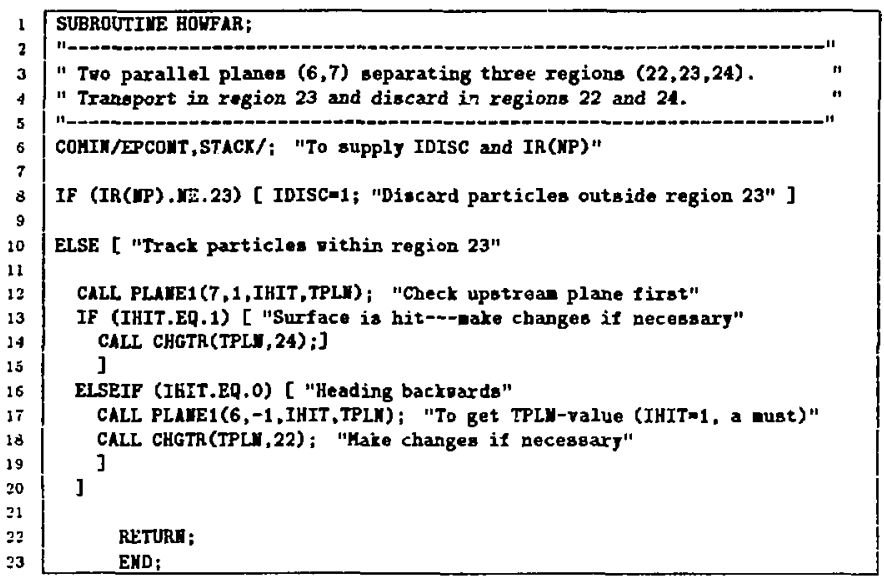

I'igure 1.2. Example HOWFAR listing corresponding to Fig. 1.1.

The first argument of PLANE1 is the number that identifies the plane and the second argument is either a 1 or a -1 depending on whelher the surface normal points $a w a y$ from or towards the current region, respectively. The third argument is IHIT and the fourth is TPLN. Although it really does not matter which of the two planes (6 or 7$)$ is checked first, we have elected to check plane 7 first (line 12) and then plane 6 (line 17) if it has been determined that the particle is moving away from plane 7 . In either case, CHGIR will be called when necessary (i.e., when IHIT=1). As shown in lines 14 and 18 , 
the first argument of CHGTR is the TPLN-value supplied by the previous call to PLANE1, whereas the second argument is the region that the particle might end up in should TPLN be smaller than USTEP.

A few things are worth noting at this time. First, if the user has any preknowledge about the radiation transport process taking place. it may be of use in selecting which plane to check first. In our example above, if it is knows that the radiation field generally moves from left to right in Fig. 1.1, it is more efficient to check plane 7 before checking 6 . The reverse is true for a beam entering along the negative $z$ direction.

Second, the statement COMIN/EPCONT, STACK/ (line 6) is needed in order to supply IDISC and IR(NP) to HCWFAR, whereis it has already been noted that COMIN/PLADTA/ is required in MAIN (in order to pass PNORM and PCOORD data to SUBROUTINE PLANE1).

Finally, although the use of PLANE1 and CHGTR may seem, at first, to be just as cumbersome as coding directly within SUBROUTINE HOWFAR, what we are attempting to demonstrate is a modular approach for creating the more complex geometries-i.e., systemizing the construction of difficult problems. As will be shown in the next section, we will gain back the efficiency of in-line coding by using macro equivalents for the geometry subprograms, and still maintain the advantage of modularity.

\subsubsection{Macro equivalents of PLANE1 and CHGTR (\$PLANE1 and \$CHGTR).}

The EGS4 Code System is written entirely in the Mortran3 language ${ }^{2}$, which has a macro facility that can be used in order to place the geometry programs directly into SUBROUTINE HOWFAR. We will not describe the Mortran3 macro facility in this note, but we will give a simple example so that the uninitiated reader can get the general idea of how things work (additional information on Mortran3 macros is provided in the EGS4 Code System documentation ${ }^{1}$ ). The example is SUBROUTINE CHGTR and its macro equivalent, \$CHGTR, which are listed in Fig. 1.3.

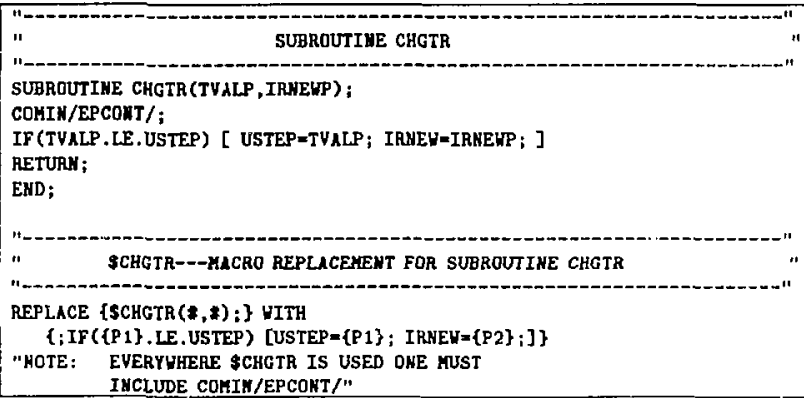

Figure 1.3. SUBROUTINE CHGTR and its macro equivalent. 
The I\$CHGTR macro can be explained as follows:

- A Mortran3 macro processor recognizes and processes specific strings.

- \$CHGIR (string1, string?) in its entirety is such a string, and it also contains the substrings, string! and string2.

- The stringl that is located at the position of the first \# is assigned to the parameter $\{\mathrm{P} 1\}$.

- The string 2 that is located at the position of the second $*$ is assigned to the parameter $\{\mathrm{P} 2\}$.

- The macro is then expanded out accordingly.

For example, if in line 14 of Fig. 1.2 the statement had read \$CHGTR (TPLN, 24) instead of CALL CHGTR(TPLN, 24), the following code would have been inserted automatically in its place during the so-called "Mortran-step" of the computer run:

\section{IF (TPLN.LE.USTEP) [USTEP=TPLN; IRNEN=24;]}

(some of the semicolons have been omitted in this discussion but, indeed, they too are considered part of strings wherever they occur, and get processed accordingly).

One therefore retains the convenience of modular coding, using statements like \$CHGTR that look similar to CALL CHGTR, without wasting computer time by forcing a call to a subprogram. Similar macros have been defined for all the EGS4 geometry subprograms listed in Section 1.2 above, including PLANE1, so that the SUBROUTINE HOWFAR example that we have been discussing in Fig. 1.1 and Fig. 1.2 can be rewritten as shown in Fig. 1.4.

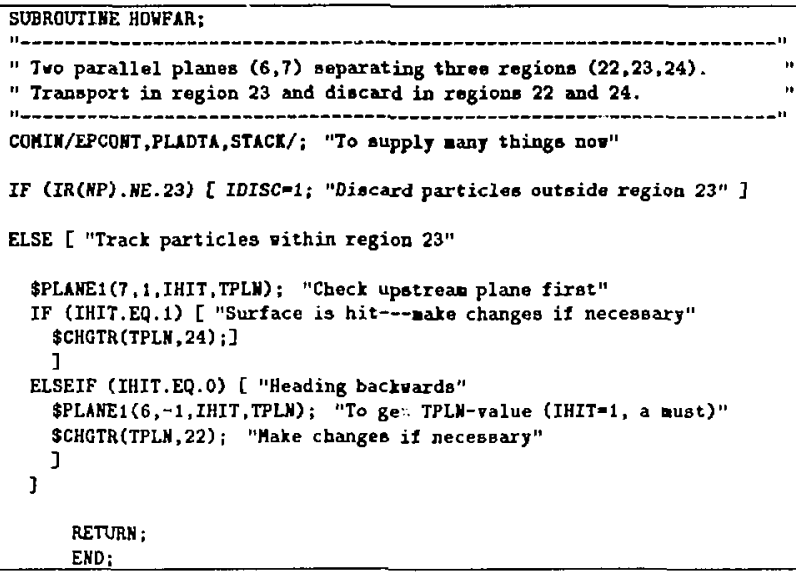

Figure 1.4. Macro equivalent of listing in Fig. 1.2. 
One point should be made at this time; namely, PLADTA has been added to the COMIN list in Fig. 1.4 (line 6), since the PNORM and PCOORD values must be passed from MAIN directly into HOWFAR, where they will now be used with the in-line code established by the macros \$PLANE1 and \$CHGTR. This raises a question: "How does one know beforehand what COMINs to include in SUBROUTINE HOWFAR when using the various geometry macros?" The answer is simple. All of the geometry macros are defined in the EGS4 file called EGS4MAC MORTRAN, and the necessary COMIN information is given at the end of each definition (similar to that shown in lines 15 and 16 at the bottom of Fig. 1.3). It goes without saying that any reference by the user to variables that are part of the EGS4 Code System must also be made available in SUBROUTINE HOHFAR (a list of variables is provided in Appendix 2 (Section A2.3) of SLAC-265).

\subsubsection{The \$PLAN2P macro.}

The HOWFAR example that we have been following can be simplified even further with the aid of \$PLAN2P*, as shown in Fig. 1.5. \$PLAN2P functions by "calling" \$PLANE1 and $\$ C H G T R$ for plane number 7 , and again for plane number 6 (but only if necessary). The simplification of SUBROUTINE HOWFAR is, indeed, apparent.

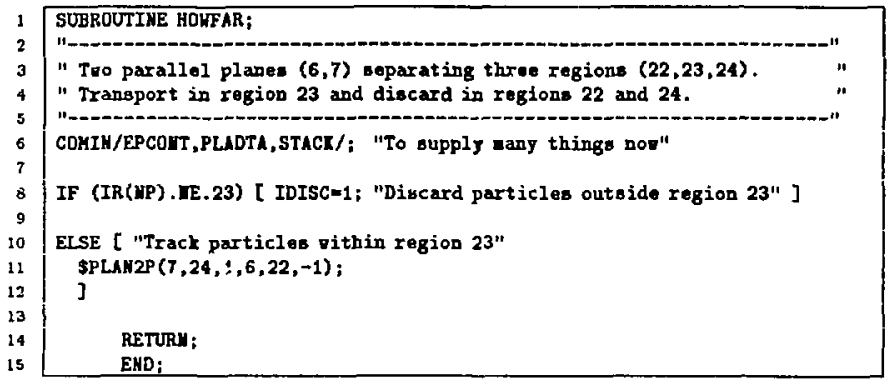

Figure 1.5. An even simpler macro equivalent of the Fig. 1.2 listing.

A sampling calorimeter example.

Essentially, the same HOWFAR can be applied to a sampling calorimeter geometry consisting of many alternating slabs of absorber and detector (i.e., a sandwich counter). For example, Fig. 1.6 could be used with NREG-1 planes (starting with plane 1) that have unit normal vectors all pointing along the $z$-direction (i.e., PNORM $=0,0,1$ ). In this exarnple, particles are discarded when they enter either region 1 or the last region (NREG) (which must be made available in SUBROUTINE HOWFAR).

- Mremonic for two parallel planes. 


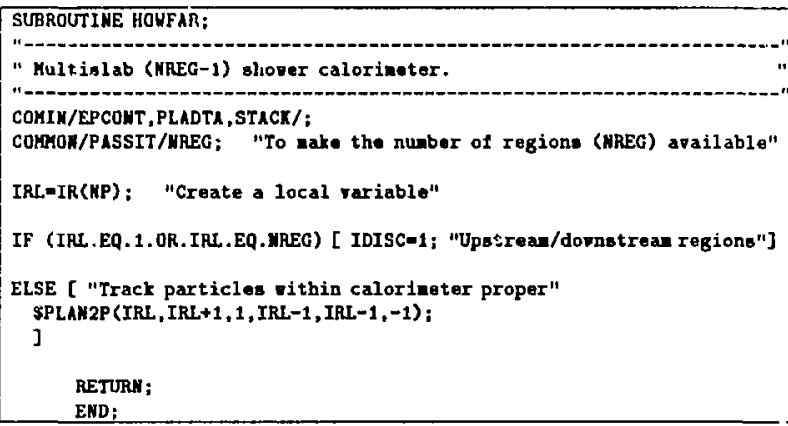

Figure 1.6. SUBRDUTINE HOWFAR for a multislab shower calorimeter.

The shower picture shown in Fig. 1.7 represents a simulation* of the MWPC detector array that was used in experiment E-137 performed at SLAC (photons are shown as dots and charged particles as solid lines). The SUBROUTINE HOWFAR that was used in this calorimeter simulation is similar to that given in Fig. 1.6. In order to simulate, as best as possible, :'uctuations due to the leakage of energy from the sides, the slab geometry was enclosed within a cylinder (e.g., see Section 1.3.5).

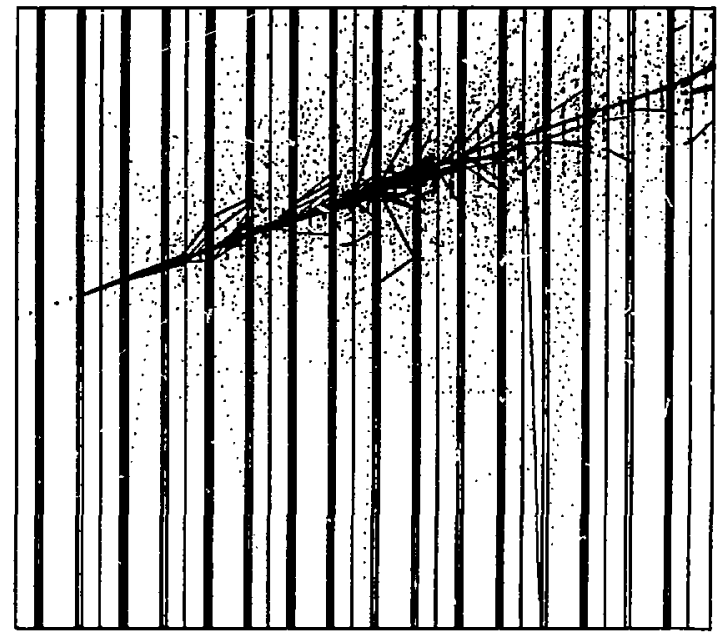

Figure 1.7. A single 5-GeV photon entering at $20^{\circ}$.

* The EGS4 User Code: UPE137. The shower graphics was done using the SHIOWGRAF package ${ }^{3}$. 
A box of slabs.

Up to this point, except for the E-137 example in the previous sect.on, we have assumed that the slab geometry is semi-infinite-i.e., laterally unbounded-although it is not difficult to include sides in our geometrical description. For example, consider an electromagnetic cascade shower counter made up of alternating slabs of material along the $\mathrm{z}$-axis (e.g., $\mathrm{Pb}$-scintillator layers) and bounded in the $\mathrm{x}$ - and $\mathrm{y}$-directions by two pairs of planes to form a right parallelepiped-i.e., a box of slabs. Since we have already pointed out that (almost) all surfaces must be checked to see if they are hit, it becomes apparent that SUBROUTINE HOWFAR consists primarily of three successive PLAN2P calls:

$$
\begin{aligned}
& \text { Two x-planes - CALL PLAN2P (IPLNX1, IRGNX1,1, IPLNX2, IRGNX2,1); } \\
& \text { Two y-planes - CALL PLAN2P (IPLNY1, IRGNY1,1, IPLNY2, IRGNY2,1); } \\
& \text { Many z-planes - CALL PLAN2P(IPLNZ1, IRGNZ1,1, IPLNZ2, IRGNZ2,-1); }
\end{aligned}
$$

where the arguments in the statements (e.g., IPLNX1, etc.) are general and refer to no particular geometrical figure shown in this note, although the 1's in the first two call staiements suggest that the normal vectors point away from the origin of coordinates. The concept should be clear.

\subsubsection{The \$PLAN2X macro.}

Figure 1.8 provides us with an example of where we both can and cannot use \$PLAN2P. Again, let us assume that the current particle position is at $P$. For simplicity, we will also discard particles if EGS transports them out of region 23. We can use \$PLAN2P with planes 8 and 9 since they are parallel to one another. Specifically, if plane 8 is hit, then 9 must be missed (and vice versa), and the parallel trajectory is parallel to both planes.

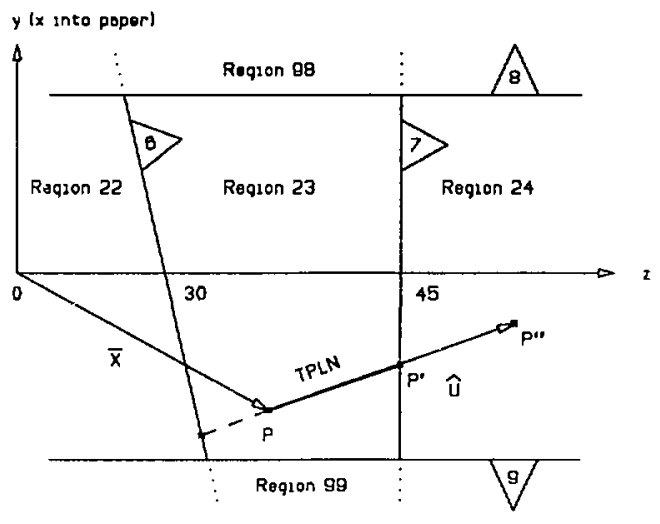

Figure 1.8. Five regions formed by a pair of parallel and a pair of crossing planes. 
However, we cannot use \$PLAN2P with planes 6 and 7 since re must always perform both sets of calculations using \$PLANE1 and \$CHGTR-i.e., we no longer have the option to ignore a set of calculations to gain efficiency. To this end, \$PLAN2 $X^{*}$ was created to take care of the crossing-plane situation. It should also be noted that the concept of semi-infinite slabs is not well-defined if we were only to have planes 6 and 7 ; hence, there is a good reason for having planes 8 and 9 in our example problem (planes 8 and 9 could have been crossing too).

The listing shown in Fig. 1.9 uses \$PLAN2X and \$PLAN2P, both of which have the same argument sequence.

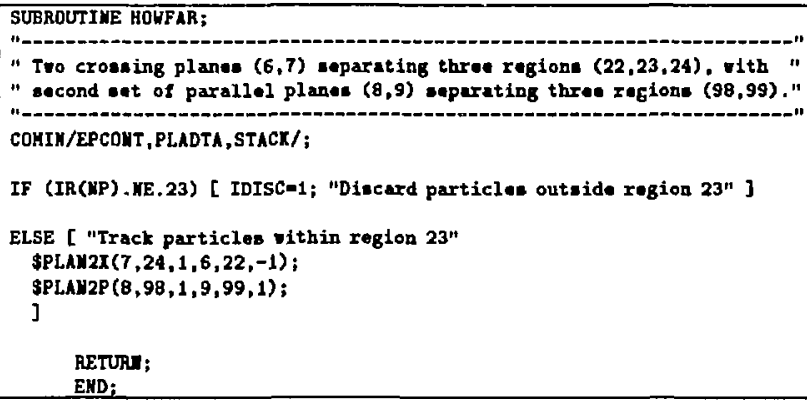

Figure 1.9. Example HOWFAk iisting corresponding to Fig. 1.8.

It is instructive to convince oneself that the following statements ar irue:

- \$PLAN2X and \$PLAN2P must both be called.

- The order in which \$PLAN2X and \$PLAN2P are called is not important.

- If one has preknowledge that the radiation field moves essentially towards one or the other parallel plane, then \$PLAN2P can be made more efficient by checking the preferred plane first.

- There is no preferred order for checking planes with \$PLAN2X.

- USTEP and IRNEW will always be properly selected.

\subsubsection{The \$CYLNDR, \$CDNE and \$SPHERE macros.}

The conic surface algorithms basically are all the same and \$CONE and \$SPHERE may be uscd in SUBROUTTNE HDWFAR in the same manner as \$CYLNDR. Therefore, only \$CYLNDR will be discussed in this note.

* Mnemonic for two crossing planes. 
The intersection of a vector with a conic surface leads to a quatratic equation, the solutions of which are both real and imaginary and correspond to actual physical sit1. ations. Figure 1.10, for exampic, shows the possible trajectories intersecting a cylinder. Trajectory (a) is for a particle traveling inside the cylinder, in which case the soluticis of interest is the positive one, as depicted by the solid line portion of (a). 'The other three trajectories correspond to particles traveling outside the cylinde:, where (d) represents an imaginary solution to the quadratic equation. Trajectory ic) is for a particle moving away from the cylinder and neither of the two negative solati 2 ns are of use io us. However, trajectory (b) corresponds to a particle moving towards ine surface and it is the smaller of the two positive solutions that ate of direct use in SUBFDUTINE HOWS:R.

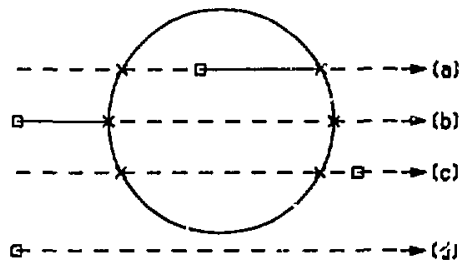

Figure 1.10. Possible trajectories intersecting a cylinder (starting and intersection points indicated by squares and crosses, respertively).

The \$CYLNDR macro (and corresponding subroutine) was designed to take all of these possibilities into account. In addition, the algorithm takes into account a difficulty that can arise due to a particle traveling very close to the cylinder surface, particulary at a glancing angle. Namely, for a given machine precision the quadratic solutions, together with the way EGS4 uses them, can result in a particle being "stepped" sideways to the surface. Albeit a very small amount, this can cause enough error in the true position of a particle to ronfuse the user's boundary tracking prograin (i.e., SUBROLTINE HOWFAR), generally with the result that the program gets caught in an infinite loop. An attempt to resolve such difficulties has been addressed by Stevenson ${ }^{5}$, and those portions of the algorit ${ }^{2} m$ involving the parameter DELCYL are a direct result of $t l^{-}:$sicudy. We will return to the question of the proper value to set DELCYL later in this section. First, let us demonstrate how one uses \$CYLNDR with a simple, but practical, example.

Consider a cylindrical target struck by an incident electron beam as shown in Fig. 1.11. The cylinder of rotation about the z-axis is identified by box 1 . There are four regions of interest-the target (region 2) and three vacuum regions upsiream, downstrean $n_{1}$ and surrounding the target. The extent of the target along the $z$-direction is determined by two end planes, identified by triangles 1 and 2 that point in the direction of the defining unit normal vectors:

$\begin{array}{lll}\operatorname{PNORM}(1,1)=0.0 ; & \operatorname{PNORM}(2,1)=0 ; & \operatorname{PNORM}(3,1)=1.0 ; \\ \operatorname{PNORM}(1,2)=0.0 ; & \operatorname{PNORM}(2,2)=0.1 ; & \operatorname{PNORM}(3,2)=1.6 ;\end{array}$

The target length, $T$, is

$$
T=\operatorname{PCOORD}(3,2)-\operatorname{PCOORD}(3,1) \quad(\mathrm{cm})
$$


(all the other PCOORD-values are zero), and the radius, $R$ is defined by

$$
R^{2}=\operatorname{CYAAD2(1)}\left(\mathrm{cm}^{2}\right)
$$

where all the quantities are (generally) defined in MAIN and passed to HOHFAR in COMIN/CYLDTA, PLADTA / in the manner that we have discussed previously. An example listing for HOHFAR is shown in Fig. 1.12. The arguments of \$CYLNDR (line 11) are similar to those of \$PLANE1, i.e., the first two are user inputs and the last two return information. The first argument is the identification number and the second argument is assigned the value of 1 or 0 , depending on whether the current position is inside or outside the cylinder, respectively. The third argument (IHIT) is assigned the value of 1 or 0 , corresponding to a hit or a miss, respectively. The intersection distance (TCYL) is of use whenever IHIT=1; Tlie remainder of the listing in Fig. 1.12 is similar to what we have described earlier.

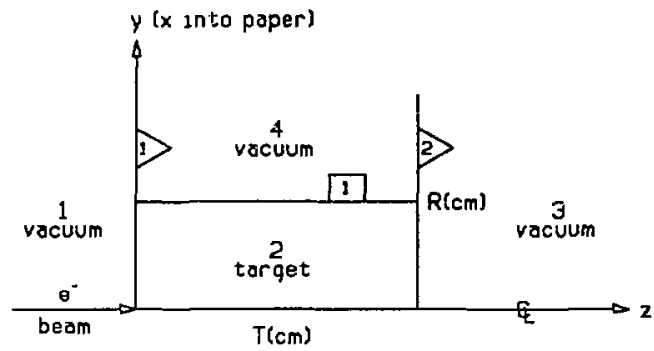

Figure 1.11. Cylinder of rotation about the z-axis bounded by two planes.

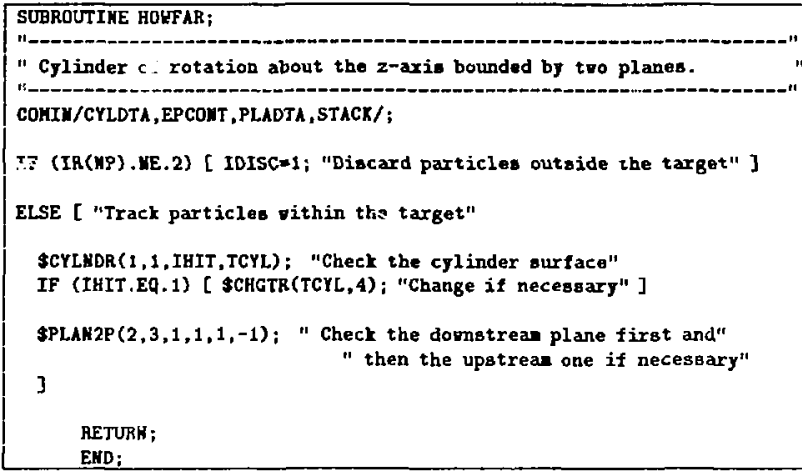

Figure 1.12. Example h. . $_{\text {AR }}$ listing corresponding to Fig. 1.11. 
The DELCYL parameter.

The "closest allowable distance to the surface", DELCYL, is actually implemented witlin the \$CYLNDR algorithm by means of a Mortran macro that recognizes and replaces the \$DELCYL template. As a practical matter, the use of a \$DELCYL-value of $1.0 \mathrm{E}-4 \mathrm{~cm}$ has been found to work satisfactorily in most situations, and this is the current value that is included in the EGS4MAC MORTRAN file-i.e., this is what the user gets by default.

For very small cylinders, the user may need to reduce the value to $1.0 E-5$ (or even $1 . \mathrm{OE}-6)$; whereas, for very large cylinders (e.g., $R \approx 10 \mathrm{~cm}$ or larger) a choice of $1.0 \mathrm{E}-3$ may be required in order to avoid the infinite-loop syndrome. The user can experiment with \$DELCYL and change its value in a dynamic way, as dictated by the particular problem at hand. Alternatively, one can select a small number for \$DELCYL, such as $1.0 \mathrm{E}-6$, and perform the entire calculation with higher precision. The AUTODBL option associated with FORTRAN compilers on IBM computers provides an easy way to accomplish this feat without having to re-code EGS4.

The easiest way for the user to change \$DELCYL is to insert an over-ridi macro at beginning of the User Code. For example,

\section{REPLACE $\{\$ D E L C Y L\}$ UITH $\{1.0 E-03 ;\}$}

would increase the value by a factor of 10 from its default value of $1.0 \mathrm{E}-04$. Similariy,

REPLACE\{\$DELCYL\} SITH \{DELCYL\}

would make it a variable that could be changed dynamically by the user in, for example, SUBROUTINE HOWFAR.

A cylinder-slab example (SLC $\mathrm{e}^{+}$target).

EGS4 has been used to determire the size and nature of the SLC positron target, beth to optimize the yield and to understand the heat problems and radiation fields*. Figure 1.13 shows an EGS4 shower generated by a single $33 \mathrm{GeV}$ electron striking the $6 X_{0}$ cylindrical target, where photons are shown as dots and charged particles as solid lines.

* The EGS4 User Code: UP33P05. 


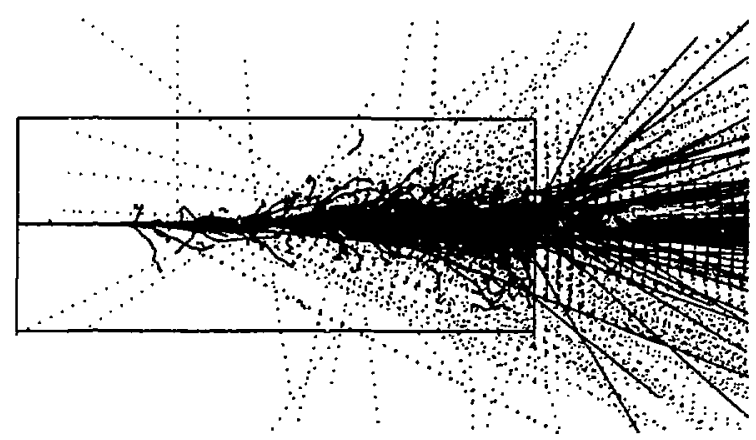

Figure 1.13. Shower produced in $6 X_{0}$ cylindriral target struck by a single 33 $\mathrm{GeV}$ electron (all particles shown).

\subsubsection{The \$FINVAL macro.}

The \$FINVAL macro is generally used in order to determine the coordinates at the point of intersection of a particle trajectory and a geometric surface-i.e., a particle's final coordinates just before passing through a boundary into a new region.

Consider, for example, the cylinder-slab geometry shown in Fig. 1.11, only this time allow particles to be transported (rather than discarded) in region 1 (e.g., a source located in region 1). A particle moving from left to right (i.e., $\hat{\mathbf{w}}>0$ ) inte-sects plane number 1 and passes into either region 2 or region $4-a$ number to be determined witb the help of \$FINVAL. The following code could be used within SUBROUTINE HOWFAR to take care of this situation.

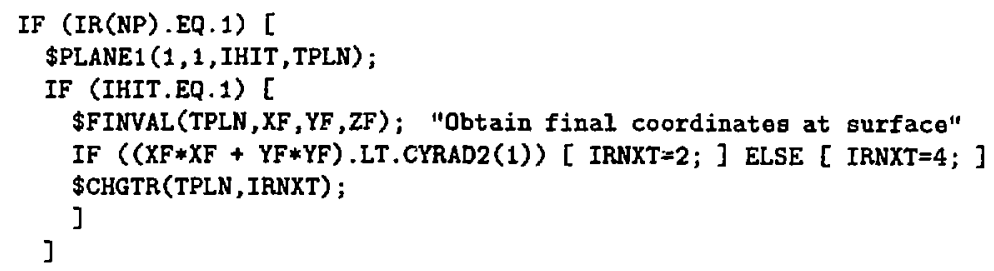

It should be noted that a square root was avoided in the IF statement alove-i.e., it is more efficient to compare "radii squared".

\subsection{COMBINNING THE ABOVE MACROS-A SIMPLE EXAMPLE}

Finally, we show how one might combine the above examples into a typical geometry. The example chosen is the target-collimator system shown, in sketch form, in Fig. 1.14. In this geometry, the electron beam strikes a $\mathrm{Pb}$ target $2 \mathrm{~cm}$ thick. This is followed by a vacuum region, and then $\mathrm{Pb}$ jaws which define the vertical beam width. The jaws are shown in view AA in Fig. 1.15. The EGS4 run of this geometry is shown in Fig. 1.16, where the shower graphics were done using the SHOWGRAF packages. An abbreviated version of User Code UPTN87-4 which created these rays is given in the Appendix. 


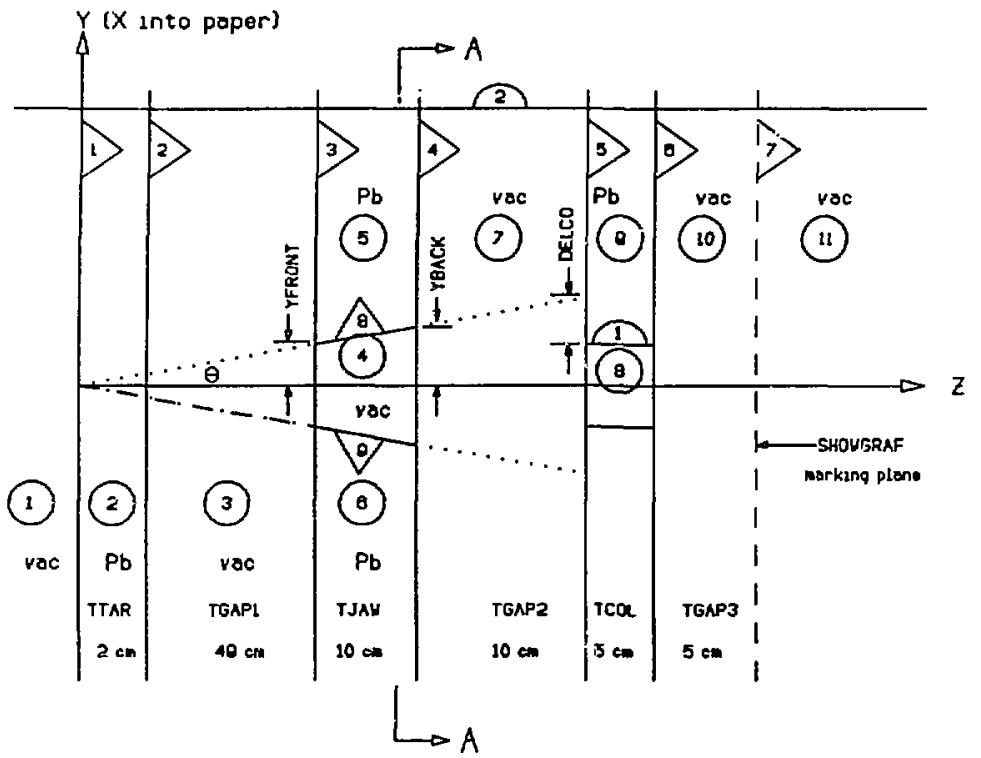

Figure 1.14. Target-collimator zystem, with vacuum between the $\mathrm{Pb}$ components. The jaws defining Region 4 are shown in a different view (AA) in Fig. 1.15. The collmmator opening defining Region 8 is circular.

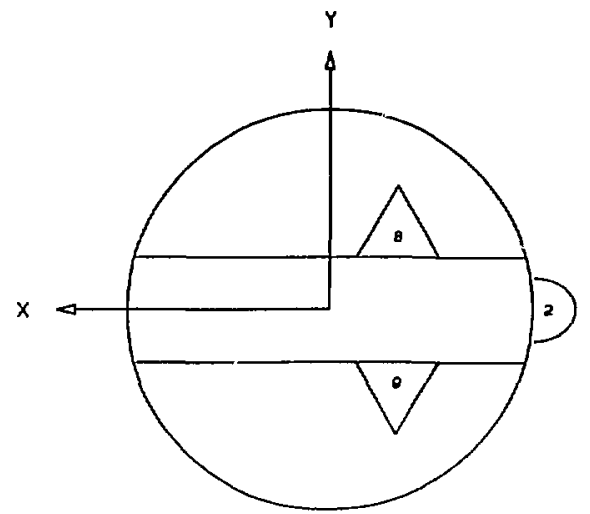

Figure 1.15. View AA of the vertical jaws in the collimator system. 


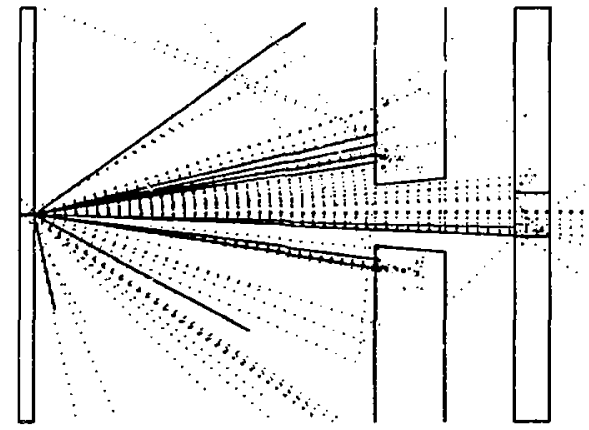

Figure 1.16. A single 1-GeV electron incident on 2-cm slab of $\mathrm{Pb}$, simulated by EGS4 using UPTN87-4 with SHOWGRAF'. Solid rays from the target location are electrons; dotted lines represent photons. 
APPENDIX

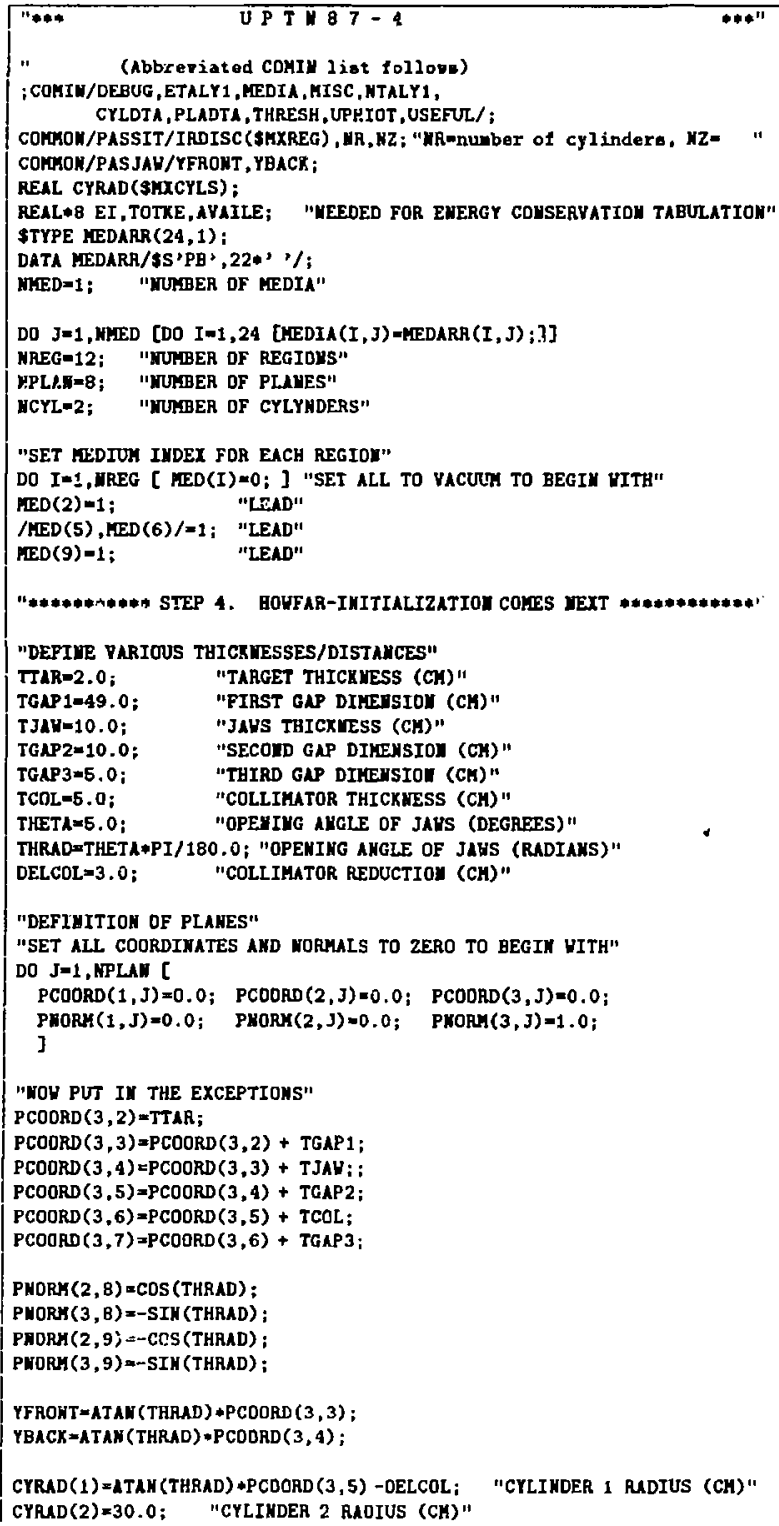


"**** STEP 6. DETERMIHATION OF IMCIDENT PARTICLE PROPERTIES \#\#"

(Abbreviated to whov only geometry-dependent itema)

IQI=-1: "INCIDENT PARTICLE"

$X I=0.0 ; Y I=0.0 ; Z I=0.0 ; \quad$ "STARTING CODRDIMATES (CH)"

UI $=0.0 ; V I=0.0 ; \quad U I=1.0$; "INCIDENT DIRECTION COSINES"

IRI=2; "EMTRAHCE REGION DEFINITIOU"

"(Initialization of AUSGAB, Shover calI, etc onitted from example)"

STOP;

END; "END OF MAIN PROGRAH"

YE

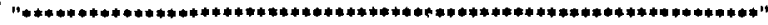

SURROUTIHE HOWFAR;

; COHIM/CYLDTA, EPCONT,PLADTA,STACK/;

CONADN/PASJAU/YFRONT, YBACK;

IRL=IR(NP); "Create a local variable"

IF(IRL.EQ.1.OR. IRR.GE.11) [ IDISC=1; "Discard regions 1, 11, or 12" ]

ELSEIF (IRL, IE. 2) [ "Region 2"

\$PLAH2P $(2,3,1,1,1,-1)$;

SCYLHOR $(2,1$, IHIT, TCYL) ;

IF (IHIT.EQ.1) [ \$CHGTR(TCYL, 12); ]

] "End of region 2"

ELSEIF (IRI.EQ.3) [ "Region 3"

SPLANE1 (3, 1 , IHIT, TPLN);

IF (IHIT.EQ.1) [

\$FIUVAL (TPLU, XF, YF,ZF);

IF (ABS (YF) ,LT,YFRONT) [ IRHXT $=4$; ]

ELSEIF (YS.GE.YFRONT) [ IRHIT-5; ]

ELSE [ IRILT=6:]

\$CHGTA (TPLW, IRHXT);

]

ELSEIF (IHIT.EQ .0) [

SPLAHE1 $(2,-1$, IHIT, TPLH);

\$CHGTR(TPLN, 2);

]

SCYLHDR( $2, \pm$, IHIT, TCYL);

IF (2uTT ER.1) [ \$CHGTR(TCYL, 12); ]

] "End of region 3"

ELSEIF (IRL.EQ.4) [ "Region 4"

\$PLAH $2 P(4,7,1,3,3,-1)$;

$\$ \operatorname{PLAK2X}(8,5,1,9,6,1)$;

SCYLXDR (2, 1, IHIT , TCYL);

IF (IHIT.EQ. 1) [ SCHGTR(TCYL, 12); ]

] "End of region 4"

ELSEIF (IRI.EQ.5) [ "Region 5"

SPIAN2P $(4,7,1,3,3,-1)$;

$\$ C Y L N D R(2,1$, IHIT, TCYL);

IF (IHIT.EQ.1) [ \$CHGTR(TCYL, 12);]

SPLANEI (8, - 1, IHIT, TPLN);

IF (IHIT.EQ , 1) [\$CHGTR (TPLN,4); ]

] "End of region 5"

ELSEIF (IRL.EQ.6) [ "Regio. 6"

$\$ \operatorname{PLAN2P}(4,7,1,3,3,-1)$;

\$CYLNDR (2, 1, IHIT, TCYL);

IF (IHIT.EQ.1) [ SCHGTR(TCYL, 12); ]

\$PLAHE1 (9,-1, IHIT, TPLH);

IF (IHIT,EQ.1) [ \$CHGTK(TPLN,4);] 
] "End of rogion 6"

ELSEIF (IRL.EQ.7) [ "Region $7 "$

\$CYLNDR (2, 1, IHIT, TCYL);

IF (IHIT.EQ.1) [ ICHGTR (TCYL, 12);]

SPLARE 1 $(5,1$, IHIT, TPLN);

IF (IHIT, EQ.1) [

SFINULL(TPLN,XF,YF, ZF):

IF ((XF*XF + YF*YF).GE.CYRAD2(1)) [ IRNXT=9;] ELSE [ IRMXT-8; ] \$CHGTR (TPLN, IRYXT);

了

ELSEIF (IHIT.EQ.0) [

SPLANE1 (4, -1, IHIT, TPLN);

SFINYLL (TPLIN, XF, YF, ZF):

IF (ABS (YF).LT.YBACX) [ IRNXT=4; ]

ELSEIF (YF.GE.YBACK) [ IRUXT=5;]

ELSE [IRMXT=6;]

\$CHGTR (TPLW, IRMXT);

了

J "End of region 7"

ELSEIF (JAL.EQ.8) [ "Region 8"

\$PLAH2P $(6,10,1,5,7,-1)$;

$\operatorname{SCYLIDR}(1,1$, IBIT, TCYL);

IF (IHIT.EQ.1) [ \$CGGTR(TCYL, 9); ]

] "End of region g"

ELSEIF (IRL.EQ.9) [ "Region 9"

SpLen $2 P(6,10,1,5,7,-1)$;

SCYLIDR (1, 0, IBIT, TCYL);

IF (IHIT.EQ.1) [ SCHGTR(TCYL,B); ]

ELSE [

SCYLDR (2,1, IHIT, TCYL):

IF (IHIT.EQ.1) [ \$CHGTR(TCIL,12); ] ]

] "End of region 9"

ELSEIF (IRL.EQ.10) [ "Region 10" SCYLMDR $(2,1$, IHIT, TCYL);

IF (IHIT.EQ.1) [ SCHGTR(TCYL., 12); ]

SPLANE1 $(7,1$, IHIT , TPLU);

IF (IHIT.EQ.1) [ \$CHGTR(TPLN, J1); ]

ELSE [ IDISC=1; "This really should not happen in a vacuun" ]

]

ELSE [ "Bad region - notify user"

OUTPUT IRL; (' IRL=',I10, ' BAD REGIOH NUIRER');

STOP;

]

RETUR: ;

END:

Y.E 


\section{REFERENCES}

1. W. R. Nelson, H. Hiravama and D. W. O. Rogers, "The EGS4 Code System", SLAC-265 (Decenber 1985).

2. A. J. Cook, "Mortran3 User's Guide", SLAC Computation Research Group Technical Memorandum Number CGTM 209 (1983).

3. R. Cowan and W. R. Nelson, "Producing EGS4 Shower Displays With Unified Graphics", Stanford Linear Accelerator report SLAC-TN-87-3 (1987).

4. A. Abashian, J. Bjorken, C. Church, S. Ecklund, L. Mo, W. R. Nelson, T. Nunamaker, P. Rassman and D. Scherer, "Search for Neutral, Penetrating, Metastable Particles Produced in the SLAC Beam Dump", presented at the Iourth Moriond Workshop on Massive Neutrinos in Particle and Astrophysics, LaPlagne, France (15-21 January 1984).

5. G. R. Stevenson, "A Cylindrical Geometry Package for HOHFAR in the EGS Electron Gamma Shower Program", CERN Internal Report Number HS-RP/TM/80-78 (November 1980); also of related interest: G. R. Stevenson and T. Lund, "A Spherical-Conical Geometry Package for HOWFAR in the EGS Electron Gamma Shower Program", CERN Internal Report Number HSRP/TM/81-30 (May 1981); G. R. Stevenson, "A 3-Dimensional Cartesian Geometry Package for HOWFAR in the EGS Electron Gamma Shower Package", CERN Internal Report Number HS-RP/TM/80-60 (September 1980).

\section{DISCLAIMER}

This report was prepared as an account of work sponsored by an agency of the United States Government. Neither the United States Government nor any agency thereof, nor any of their cmployees, makes any warranty, express or implied, or assumes any legal liability or responsibility for the accuracy, compleleness, or uselulness of any information, apparatus, product, or process disclosed, or represents that its use would not infringe privately owned rights. Reference herein to any specific commercial product, process, or service by trade name, trademark, manufacturer, or otherwise does not necessarily constitute or imply its endorsement, recommendation, or favoring by the United States Government or any agency thereof. The views and opinions of authors expressed herein do not necessarily state or refiect those of the United States Government or any agency thereof. 